\title{
Variation between Initial and Final Diagnosis as A Predictor of Adverse Obstetric Outcome among Post- Natal Women at Bungoma Hospitals, Kenya
}

\author{
David Nandebe Wafula, Bernard Wesonga, Maximilla Wanzala, Nathan Shaviya, and Rose A. M. \\ Nyang'au
}

\section{ABSTRACT}

Despite diagnosis being an important part of clinical or medical consultations, the diagnosis might fail leading to adverse effects. This is a global problem, where developed and developing countries go through. In sub-Saharan Africa, variations between initial diagnosis and final diagnosis lead to diagnostic errors with high maternal mortalities. In Kenya, a lot of measures have been put in place but still, variation in diagnosis appear to have become rampant. Bungoma county is one of the counties with a high mortality ratio, especially for pregnant women attributed to the variation between initial and final diagnosis. Therefore, it was crucial to investigate the variation between initial and final diagnosis in relation to obstetric outcomes at hospitals in Bungoma County. The cross-sectional research design was used (Bungoma and Webuye hospitals). Systematic sampling was used to obtain 384 respondents after proportionate allocation to each hospital, and purposive sampling to select 8 health care workers as key informants. Data was collected using a structured questionnaire and an interview guide. The pre-test was done with validity established through crosschecking and reliability calculated using the Cronbach method $(0.89)$. Using a statistical package for social sciences version 25 , descriptive and inferential statistics was run where chi-square and odds ratio was used to determine the influence between variables, significance and prediction. The study revealed a variation between initial diagnosis and the final diagnosis was $\mathbf{2 0 . 8 \%}$ while diagnostic errors were significant predictors of obstetric outcomes among post-natal mothers at level five with a p-value of 0.045 at a significance of $5 \%(P=0.045)$. Demographic characteristics showed no relationship with obstetric outcomes $(P=0.54>0.05)$. Matched diagnostic had no variations $(\mathrm{N}=327, \mathrm{M}=1.00, \mathrm{SD}=\mathbf{0 . 0 0 0})$; while unmatched diagnostic had variations $(\mathrm{N}=327, \mathrm{M}=1.82, \mathrm{SD}=.384)$. There was a relationship between diagnostic errors and obstetric outcome $\left(\chi^{2}(1)=251.86, p<.001\right)$. An association between diagnostic error with unsafe obstetric outcomes was significant at the odds ratio of 2.03 (OR $2.03,95 \%$ CI 1.31-2.16). The study demonstrates that a correct diagnosis is a viable strategy in preventing unsafe obstetric outcomes and by extension minimizing morbidity and mortality among pregnant women. The study concluded that there was a variation between initial diagnosis and final diagnosis which had an adverse obstetric outcome. it was recommended to build capacities for the health workers in order to address increased diagnostic errors.

Keywords: Adverse obstetric outcome, bungoma, diagnosis, matched and unmatched errors, pregnancy, variation.
Submitted : December 7, 2021

Published : January 4, 2022

ISSN: $2593-8339$

DOI: $10.24018 /$ ejmed.2022.4.1.1170

D. N. Wafula*

Kenya Medical Training College, Nairobi, Kenya.

(e-mail: nandebedavid@yahoo.com) B. Wesonga

Masinde Muliro University of Science and Technology, Kakamega, Kenya.

(e-mail: bwesonga2@gmail.com) M. Wanzala

Masinde Muliro University of Science and Technology, Kakamega, Kakamega County, Kenya.

(e-mail: mwanzala@mmust.ac.ke)

N. Shaviya

Masinde Muliro University of Science and Technology, Kakamega, Kakamega County, Kenya.

(e-mail: nshaviya@mmust.ac.ke)

R. A. M. Nyang'au

Kenya Medical Training College,

Nairobi, Kenya.

(e-mail: rosemogoi@gmail.com)

*David Nandebe Wafula

\section{INTRODUCTION}

Despite an impressive evolution in diagnostic technology, the disparity between clinical diagnosis and autopsy is not declining [1]. Diagnosis is meant to provide a reliable clinical condition of the client [2]. The deviation of the final diagnosis from the initial diagnosis which is an unmatched diagnosis lead to variations [2]-0 The right diagnosis has a positive health outcome in most cases. However, health care facilities lack the tools and strategies for measuring diagnostic safety and most have not integrated diagnostic error into their existing patient safety programs [4]. In America, reports indicate misdiagnosis as a significant problem nearing and surpassing mortalities from road traffic accidents and therefore a significant problem [5]. Unsafe health care has been recognized as a global challenge and much has been done to understand the causes, consequences and potential solutions to this problem [6]. There is a $71.4 \%$ association between cognitive biases and therapeutic or management errors [7]. Variations in diagnostic errors arise from both 
cognitive biases and knowledge deficits on a particular medical problem [8]. Since patients expect health care providers to be competent to diagnose, plan manage, carry out practical procedures, errors are prone to occur [9]. About 5\% of the adults are misdiagnosed in outpatient, while $50 \%$ of those misdiagnosed end up with unsafe outcomes [10]. In the Philippines, misdiagnosis among obstetric providers is 2.96 times more likely to cause an adverse obstetric outcome for women with missed complications [11]. Therefore, [12] agree that there is a high degree of uncertainty of diagnosis that comes from preventable harms. Diagnostic errors contributed to as many as $70 \%$ of adverse health outcomes [12]. Reflection on initial diagnosis before making the final diagnosis minimizes diagnostic errors [13].

Despite ANC being a success story in Africa as observed by World Health Organization [14], the maternal mortality rate is still high not only in third world countries but also in Kenya. The antenatal period of pregnancy presents essential interventions that may be vital to pregnant women health, with a safe outcome [15].

In Kenya, complications arising from pregnancy and puerperium are leading causes of inpatient morbidity and mortality in females [16]. The average maternal mortality is at 362 deaths per 100,000 lives, due to diagnostic errors [17]; yet the recommended average maternal mortality should reduce to 144 deaths per 100,000 live births. It is known that at least $15 \%$ of pregnant women in Kenya develop lifethreatening pregnancy complications [18], 0. A total of 936 cases of misdiagnosis have been lodged in courts for legal redress since 1997. Initial diagnosis is the first considered diagnosis that informs the initiation of the first phase of management of the patient while the discharge diagnosis involves chronological analysis through investigative and interpretational confirmatory diagnosis. In Kenya [19] over $75 \%$ of deaths are borne from diagnostic errors. This is a result of diagnostic errors that mask the magnitude of complications. Moreover, the World Bank reported that most of the deaths occur within the first 24 hours before and after delivery; being the most critical time [19].

Bungoma County still records a maternal mortality ratio of 382 maternal deaths per 100,000 live births [20]. These previous studies have shown that the maternal mortality ratio is still high; however, they are not clear whether it is promoted by diagnostic errors among pregnant women who receive obstetric services. It was against this backdrop that the study was executed to investigate the diagnostic errors as predictors of obstetric outcomes at level five hospitals in Bungoma County.

\section{Problem Statement}

The matching initial and final diagnosis is an important assurance of a safe obstetric outcome. according to previous studies. This is not true as per the global records of diagnostic errors in all spheres of health complications. Therefore, medical errors have been sufficiently investigated as the main contributors to adverse health outcomes. Also, the variation of the diagnostic errors has been established as one of the main contributors of unsafe obstetric outcomes, which have been given little attention as compared to medical errors. In sub-Saharan Africa, the maternal mortality rate is on the rise, especially during deliveries. It is clearly known whether mismatch of the initial and final diagnosis is the cause of high maternal mortality. The current status of maternal mortality in Kenya is high $(362 / 100,000)$. In Bungoma County, the maternal mortality rate is even higher than the Kenyan average $(382 / 100,000)$. Thus, in Kenya, there is no clear information on the increased maternal mortality ratio. It is not known whether variations in diagnosis are the diagnostic errors that lead to the increased probability of adverse obstetric outcomes.

The scanty information on the variation of diagnosis (initial and final) does not provide tangible information on whether it contributes to adverse obstetric outcomes. It was important thus to provide data on the variation of diagnosis and obstetric outcomes. This study was important as it helped identify the linkage between variation in diagnosis (initial and final) in promoting safe obstetric outcomes. The purpose was to investigate the variation between initial and final diagnosis as a predictor of adverse obstetric outcomes among post-natal women at level five hospitals in Bungoma County, Kenya.

\section{CONCEPTUAL FRAMEWORK}

\begin{tabular}{|c|c|c|}
\hline Independent & Proximate & Dependent \\
\hline $\begin{array}{l}\text { Diagnostic errors } \\
\text { Variation } \\
\text { between initial } \\
\text { and final } \\
\text { diagnosis }\end{array}$ & $\begin{array}{l}\text { Wrong and } \\
\text { untimely } \\
\text { Intervention }\end{array}$ & $\begin{array}{l}\begin{array}{l}\text { Adverse } \\
\text { obstetric } \\
\text { outcomes } \\
\text { (unsafe) }\end{array} \\
\text { Foetal/neonatal } \\
\text { complications } \\
-\quad \text { Asphyxia } \\
-\quad \text { IUFD } \\
-\quad \text { Foetal distress } \\
\text { Mother } \\
-\quad \text { Fits } \\
-\quad \text { DVT } \\
-\quad \text { Maternal } \\
\text { distress }\end{array}$ \\
\hline
\end{tabular}

Fig. 1. Self-constructed conceptual framework.

\section{Materials AND Methods}

The study was carried out in Bungoma County, focusing on level five hospitals (Bungoma and Webuye). It has a surface area of over $3,000 \mathrm{~km} 2$ with an estimated population of 1.7 million [21]. The research design was cross-sectional. The study population was made up of the women admitted in the postnatal wards [22] with pregnancy complications; Webuye hospital - 5,510 admissions in maternity per year whereas Bungoma hospital - 6,730 admissions in maternity per year. There are 12,240 admissions in maternity wards per year, which formed the study. A mixed sampling method was used - systematic sampling was used to get the post-natal mothers where every kth number was picked from the list of women on discharge. Before establishing the kth value, the starting value was randomly selected in excel using the random function. The post-natal mothers provided their records for verification through content analysis during the interview. Purposive sampling was used to pick the health workers who worked in antenatal care and maternity units who had vast experience with the areas of concern as key informant interviewees (consultant obstetrician gynaecologist, medical officers, reproductive health clinical 
officers and nurses working in maternity). The sample size was determined using Fisher's formula [23]. Using proportions, Bungoma County Referral Hospital had 211 participants whereas 173 were allocated to Webuye Hospital. Health care providers were purposively sampled for key informant interviews in either of the hospitals that depended on availability. A questionnaire, checklist and interview were used for data collection. Reliability was determined using the split-half test and Cronbach coefficients of 0.891 was obtained. Validity was achieved by cross-checking with the supervisors. Quantitative data were presented in frequencies tables, mean; standard deviation; inferential statistics were presented in chi-square and logistical regression. Qualitative were presented in verbatim.

The determination of variation between the initial diagnosis and the final diagnosis was assumed as an important predictor of obstetric outcomes (safe or unsafe). Therefore, the sampled women personal medical records were used to ascertain whether the initial diagnosis and final diagnosis matched or did not match. More, the study was interested in the obstetric outcome from both the matched and the unmatched diagnoses.

Ethical clearance was obtained from Masinde Muliro University of Science and Technology; Institutional Ethics and Review Committee (IERC), Bungoma County Research Committee and National Commission for Science, Technology, and Innovation (NACOSTI). The researcher had to maintain the anonymity and confidentiality of the participants.

\section{RESUlts}

A. Variations between Initial Diagnosis and Final Diagnosis at Level Five Hospitals

From the 327 investigated cases, it was determined that matched diagnosis was $72.4 \%$ (237). The unmatched diagnosis was $27.6 \%$ (90). It was further determined that, out of $72.4 \%$ of the matched diagnosis, $70.6 \%$ (231) had a safe obstetric outcome while $1.8 \%$ (6) had an unsafe obstetric outcome. The unmatched diagnosis, on the other hand, had $6.8 \%$ (22) safe obstetric outcomes while 20.8\% (68) had unsafe obstetric outcomes. The findings are presented in Table I.

TABLE I: MATCHED AND UNMATCHED DiAgNOSIS AS PREDICTORS OF OBSTETRIC OUTCOMES

\begin{tabular}{cccc}
\hline \hline $\begin{array}{c}\text { MCH Booklet } \\
\text { diagnosis content } \\
\text { analysis }\end{array}$ & \multicolumn{2}{c}{ Obstetric Outcome } & \\
\cline { 2 - 4 } & $\begin{array}{c}\text { Safe } \\
\text { Outcome }\end{array}$ & $\begin{array}{c}\text { Unsafe } \\
\text { Outcome }\end{array}$ & Total \\
\hline \hline Matched & 231 & $6(1.8 \%)$ & 237 \\
diagnosis & $(70.6 \%)$ & & $(72.4 \%)$ \\
Unmatched & $22(6.8 \%)$ & $68(20.8 \%)$ & 90 \\
diagnosis & 253 & $74(22.6 \%)$ & 327 \\
Total & $(77.4 \%)$ & $74 \%)$ \\
\hline \hline
\end{tabular}

$\overline{\mathrm{[MCH}}=$ Mother and Child Health Booklet, Matched diagnosis = initial and final diagnosis was correctly done; Unmatched = either initial diagnosis or final diagnosis was incorrectly done; $n=327] \mathrm{N}=327$.

The key informant interviews corroborated the findings in Table I.

1.... The extent of diagnostic errors seems to be on the increase and is generally associated with the present training of health care providers, lack of basic diagnostic equipment for most health facilities during focused antenatal care such as ultrasound.

2. ... Any single diagnostic error exposes a pregnant woman and her baby to a high risk of complication and possible death.

3. ... Correct diagnosis improves the pregnancy outcome, lowers the cost. Initial diagnosis and final diagnosis are up to almost $10 \%$ due to poor history taking and irrelevant investigations.

4. ... With diagnostic error, a client is given a wrong intervention leading to an effect on the pregnancy. A common misdiagnosis occurs in pre-eclampsia where it is confused for hypertension.

The variation of the initial diagnosis and final diagnosis were computed using the matched diagnosis and the unmatched diagnosis. Matched diagnosis indicated that there was consistency between initial diagnosis and final diagnosis while unmatched indicated there was a discrepancy and not related at all between initial diagnosis and final diagnosis. The matched diagnosis was coded and the unmatched diagnosis was coded 2. According to Table II, the matched diagnosis $(N=327, M=1.00, S D=.000)$ had no variation between initial diagnosis and final diagnoses. However, the unmatched diagnosis $(N=327, M=1.82, S D=0.384)$ revealed that there was a variation in diagnosis.

TABLE II: VARIATIONS OF MATCHED AND UNMATCHED DIAGNOSIS

\begin{tabular}{ccc}
\hline \hline Variations & Mean & Std. Deviation \\
\hline \hline Matched & 1.00 & 0.000 \\
Unmatched & 1.82 & 0.384 \\
Total & 1.23 & 0.419 \\
\hline \hline
\end{tabular}

[Matched diagnosis=initial and the final diagnosis was in agreement; unmatched diagnosis=initial diagnosis was not in agreement with a final diagnosis] $\mathrm{N}=327$.

\section{B. Relationship between Diagnostic Errors as Predictors of Obstetric Outcome}

The Chi-square test of independence was computed at the 0.05 significance level to determine the prediction of unsafe obstetric outcomes from diagnostic errors. Table III presents the findings that the diagnostic errors (matched and unmatched diagnosis) were predictors of adverse obstetric errors $\left(\chi^{2}(1)=251.86 \mathrm{a}, \mathrm{p}<0.001\right)$.

\begin{tabular}{|c|c|c|c|}
\hline \multirow{2}{*}{ Diagnostic Errors } & \multicolumn{2}{|c|}{ Obstetric outcome } & \multirow{2}{*}{ Statistics } \\
\hline & Safe & Unsafe & \\
\hline Matched & 231 & 6 & \multirow{3}{*}{$\begin{array}{c}\chi^{2}(1)=251.86^{\mathrm{a}}, p= \\
0.000\end{array}$} \\
\hline Unmatched & 22 & 68 & \\
\hline Total & 253 & 74 & \\
\hline
\end{tabular}

$\overline{\mathrm{a}=0 \text { cells }(0.0 \%) \text { have expected count less than } 5 \text {. The minimum expected }}$ count is 20.37; Matched diagnosis = initial and final diagnosis were agreeing; Unmatched $=$ either initial diagnosis or final diagnosis or both were not agreeing; Safe outcome = pregnancy had no complication; unsafe outcome = pregnancy had complications; $\mathrm{n}=327]$

The bivariate analysis was performed to establish the association between diagnostic errors (matched and unmatched diagnosis) and obstetric outcomes (safe and unsafe) among post-natal women. Unsafe outcomes were determined by the presence of complications in the patient record. The study found that providers who misdiagnosed or made diagnostic errors during the antenatal care visits, outpatient department and admission were 2.03 times more 
likely to have patients with a complication compared with providers who did not misdiagnose (OR 2.03, 95\% CI 1.31 $2.16, \mathrm{p}=0.046)$. The findings are presented in Table IV.

TABLE IV: PREDICTORS OF OBSTETRIC OUTCOMES

\begin{tabular}{ccccc}
\hline \hline \multirow{2}{*}{$\begin{array}{c}\text { Diagnostic } \\
\text { Errors }\end{array}$} & \multicolumn{2}{c}{$\begin{array}{c}\text { Obstetric } \\
\text { outcome }\end{array}$} & $\begin{array}{c}\text { Odds ratio } \\
(95 \% \mathrm{CI})\end{array}$ & $p$-value \\
\cline { 2 - 3 } & Safe & Unsafe & & \\
\hline Matched & 231 & 6 & $2.03(1.31$, & 0.046 \\
Unmatched & 22 & 68 & $2.16)$ & \\
Total & 253 & 74 & 0.040 \\
\hline \hline
\end{tabular}

The computed linear regression established that the variation of diagnostic errors $(\mathrm{F}(1,326)=659.160, \mathrm{p}=.045)$ significantly predicted unsafe obstetric outcomes by $4.5 \%, \mathrm{p}=0.045$.

\section{DISCUSSION}

\section{A. The Variations between Initial Diagnosis and Final Diagnosis}

The investigation on the variation between initial and final diagnosis among post-natal women in Bungoma County teaching and referral hospital showed that the matched diagnosis did not vary. The variation from the unmatched diagnosis indicated that the initial and final diagnosis was not agreeable. Therefore, the unmatched diagnosis was highly varied which increased the risk of unsafe obstetric outcomes among pregnant women. It was a known fact that the way pregnant women are handled at the antenatal care clinic and maternity was important in this study of diagnostic errors as a predictor of the obstetric outcome.

This study established that pregnant women were being handled well at the facility, where the healthcare workers at antenatal care shared antenatal information concerning the pregnancy progress, which was important for birth preparedness. But, still, maternal mortality was 382 deaths per 100,000 live births. What made it a continuous problem in health facilities in Bungoma County? It was determined that most facilities were ill-equipped to effectively diagnose from the first time of consultation where the initial diagnosis was essential in planning for the next course of action. The protocols for diagnosis and subsequent treatment of a pregnancy-related condition were poor thus promoting increased diagnostic errors. The results from the study showed that initial diagnosis and final diagnosis had a standard deviation of 0.387 whereas with this variation when projected to pregnancy outcomes there were $20.4 \%$ unsafe pregnancy outcomes resulting from these diagnostic errors. This finding tends to emphasize and support the findings [24] that showed that disparities between initial diagnosis and final diagnosis stood at 57\%. [24] Alluded to the gap being present in the obstetric care department dealing a significant effect to not only pregnant women but also on neonates.

In clinical practice, achieving a low degree of variability between initial diagnosis and discharge diagnosis is essential. Making accurate diagnoses helps in subsequent decision making especially in reproductive health where time is of the essence [25]. The results of the study showed that initial diagnosis had a significant variation to the final diagnosis which tends to agree with a study in India that gave $18.4 \%$ prevalence disagreement on clinical autopsy [1], while [24] research finding gave a variation to the tune of $57 \%$. This finding, therefore, showed that the discrepancies between initial diagnosis and final diagnosis eventually affected the management of the patient and subsequent outcomes. It was, therefore, pertinent that an initial diagnosis was correct for effective, quality and timely intervention.

A Kenyan retrospective study by [26], stated that complications arising from pregnancy and puerperium have been leading to increased morbidity and mortality in females and neonates. Direct causes of maternal mortality resulting from obstetric complications of pregnancy, labour and puerperium including interventions or any after-effects of these events. The delay in receiving adequate and or correct emergency care from the initial misdiagnosis of a pregnant woman contributes to the underlying causes of maternal mortality and morbidity. Timeliness of interventions including correct early initial diagnosis is imperative if adverse maternal outcomes are to be averted. About the USA, [10] opined that about five per cent of the adult population were misdiagnosed while they sought outpatient, services.

[15] Avers that the purpose of focused antenatal care (FANC) is to optimize maternal and foetal health, provide maternal and foetal screening (initial diagnosis), make medical and social interventions where necessary and ultimately improve the outcomes of the pregnancy. However, the study reveals that there was nobody to interpret the results at $7 \%$ during FANC visits and $\& 7.3 \%$ at OPD. The study also revealed that diagnostic errors generally ranged from $7.0 \%$ to $18.7 \%$ cumulatively. FANC visits alone without an initial diagnosis that matches with discharge diagnosis is insufficient to alter maternal outcomes. It was therefore noted that without incorporating good diagnostic management practices, FANC visits alone may not yield safe pregnancy outcomes. This study is also collaborated by a study of gap analysis between initial diagnosis and final diagnosis [1] where the study compared clinical diagnosis and autopsy findings. In this study, it was noted that there were incidences where the initial diagnosis was missing in $43 \%$ of the cases. High prevalence rates observed in the study seem to suggest corresponding unsafe outcomes and a subsequent increase in maternal morbidity and mortality.

\section{B. Relationship between Diagnostic Errors as Predictors of Obstetric Outcomes}

Singh's theory [27] states that diagnostic errors may result in adverse outcomes and that diagnostic errors misdirect interventions that may eventually result in an adverse pregnancy outcome. The study finding revealed that indeed there were diagnostic errors that influenced obstetric outcomes at Bungoma level five referral hospitals. Chisquare performed showed that there was an association between diagnostic errors and pregnancy outcomes. These results are consistent with a study done [28] in Mozambique, where major diagnostic errors were associated with up to $58 \%$ adverse outcomes and these outcomes resulted in $70 \%$ mortalities. These findings are further reinforced [25] who acknowledges that timeliness of interventions including correct early initial diagnosis was imperative if adverse maternal outcomes were to be averted.

This study shows that unnecessary investigation, delayed diagnosis and wrong diagnosis contributed to increased diagnostic errors. This was evident that health care providers tend to pay little attention to patients' conditions and order unnecessary investigations [11] Furthermore, some health 
practitioners waste a lot of time to make a diagnosis especially during the first antenatal care visit, which may magnify the underlying condition hence leading to the wrong diagnosis. It was also established that diagnostic errors were a statistically significant predictor of unsafe obstetric outcomes. However, with matched diagnosis, the chances of unsafe obstetric outcomes are very negligible. Health practitioners need to endeavour to mitigate diagnostic errors in order to promote safe obstetric outcomes. [5] Diagnostic errors have a significant bearing on the totality of patients or clients care. Diagnostic errors impact negatively the quality of health care of the patient or client. The impact can be in the form of physical, spiritual, psychological, and social trauma. Complications arising from pregnancy and puerperium are the leading causes of morbidity and mortality in women and the timeliness of interventions during diagnosis is pertinent for the subsequent reduction in mortalities [18].

To examine the relationship between diagnostic errors and obstetric outcomes, logistic regression was done and it was found that diagnostic errors predicted obstetric outcomes with diagnostic errors having 2.03 more likely to result in an adverse obstetric outcome. This finding tended to agree with a study done in the Philippines where the Odds ratio was 2.96 . However, the study in the Philippines was a simulation and was mainly centred on the health care workers whereas the study in Bungoma mainly was directed at those affected women who were pregnant. Any form of diagnostic errors, therefore, that can be minimized may help in reducing the probability of an adverse outcome and promoting safe pregnancy outcomes. To illustrate the magnitude of the adverse effects of diagnostic errors, a study was carried at Moi Teaching and Referral Hospital [26] the hospital record audit showed that $51 \%$ of the neonatal mortalities occurred and $64 \%$ of maternal deaths did occur and this shows the morbidities and mortalities that occurred during and at birth which may have been influenced by diagnostic errors. These views were reinforced by the views expressed by [5] that misdiagnosis causes more fatalities than road traffic accidents in the United States of America

History taking and physical examination form the basis of the diagnostic process in a workstation. However, in this error of technology, [29], asserts that the workstation provides the context in which the diagnostic process occurs. And this takes us to the investigation part. In this study, it was noted that unnecessary investigation and lack of interpretation was an issue that contributed to the diagnostic error and therefore influenced the pregnancy outcomes. It's worth noting that in some instances backed by the key informant interview, it has been suggested that with some point of use innovative equipment, instant diagnosis can be made and therefore improve initial diagnosis. Towards the same direction, [30] suggests that minimizing diagnostic errors in an acute setting like obstetrics require prudent use of technology as a point of use ultrasonography

Direct causes of maternal mortality result from obstetric or pregnancy complications [16]. the complications are conditions which if diagnosed early the correct interventions were undertaken. Early diagnosis especially initial diagnosis is therefore essential in minimizing diagnostic errors that are important in promoting pregnancy outcomes, whereas delayed, missed, absent, the wrong diagnosis with unnecessary investigations and wrong interpretations do influence pregnancy outcomes adversely.

\section{CONCLUSION}

The unmatched diagnosis contributed to the high burden of the unsafe obstetric outcome. The matched diagnosis had no variation. Therefore, the variation between the initial diagnosis and the final diagnosis was a statistically significant predictor of obstetric outcome. The unnecessary investigation, delayed diagnosis and wrong diagnosis contributed to increased diagnostic errors among the pregnant women in level five hospitals in Bungoma County. Moreover, diagnostic errors were 2.03 times more likely to influence the adverse obstetric outcome as compared to safe diagnoses.

\section{RECOMMENDATION}

Ministry of Health and the County Government of Bungoma to consider the introduction of precise and simple diagnostic technology equipment such as handheld ultrasound for obstetric use including training of health workers in their application. There should be rapid and multiple consultations among health care workers during health care provision to pregnant mothers to improve the accuracy of the diagnosis.

\section{ACKNOWLEDGEMENT}

I acknowledge the almighty God who has been my pillar throughout this study. I owe gratitude to all the women and health workers who were my respondents during the study. I applaud the research assistants in Bungoma and Webuye Hospitals; Inviolata Makhanu, Fatuma, Pamela Pwaipwai and Leonard Lusenaka who tirelessly worked hard during COVID 19 pandemic but ensured the study ran smoothly. I thank my supervisors Dr Benard Wesonga, Dr Maximilla Wanzala and Dr. Nathan Shaviya for their relentless support and commitment towards making sure I finish the work on time through their diligence supervision. I wish to salute Mr Paul Kem and all the staff of the following hospitals for their support, Bungoma hospital, Webuye Hospital and Kimilili Sub County Hospital. Finally, I also wish to appreciate my family and classmates for their encouragement and support.

\section{REFERENCES}

[1] Vougiouklakis T, Fragkouli K, Mitselou A, Boumba V. A comparison of the provisional clinical diagnosis of death with autopsy findings. Rom J Leg Med. 2011; 19(3): 177-82.

[2] Das J, Holla A, Das V, Mohanan M, Tabak D, Chan B. In urban and rural India, a standardized patient study showed low levels of provider training and huge quality gaps. Health affairs. 2012; 31(12): 2774-84.

[3] Bruno MA, Walker EA, Abujudeh HH. Understanding and confronting our mistakes: the epidemiology of error in radiology and strategies for error reduction. Radiographics. 2015; 35(6): 1668-76.

Jutel A. Sociology of diagnosis: a preliminary review. Sociology of health \& illness. 2009; 31(2): 278-99.

[4] Singh H, Sittig DF. Advancing the science of measurement of diagnostic errors in healthcare: the Safer Dx framework. BMJ Quality \& Safety. 2015; 24(2): 103-10.

[5] Harolds JA. Quality and Safety in Health Care, Part V: Introduction to: Crossing the Quality Chasm. Clinical Nuclear Medicine. 2015; 40(12): 959-61. 
[6] World Health Organization. Maternal mortality. [Updated November 2015]. Available from: http://www.who.int/mediacentre/factsheets/fs348/en/ [cited 15 March 2016].

[7] Saposnik G, Redelmeier D, Ruff CC, Tobler PN. Cognitive biases associated with medical decisions: a systematic review. BMC Medical Informatics and Decision Making. 2016; 16(1): 1-4.

[8] Norman GR, Monteiro SD, Sherbino J, Ilgen JS, Schmidt HG, Mamede $\mathrm{S}$. The causes of errors in clinical reasoning: cognitive biases, knowledge deficits, and dual-process thinking. Academic Medicine. 2017; 92(1): 23-30.

[9] Nathan RO, Swanson JO, Swanson DL, McClure EM, Bolamba VL, Lokangaka A, Pineda IS, Figueroa L, López-Gomez W, Garces A, Muyodi D. Evaluation of focused obstetric ultrasound examinations by health care personnel in the Democratic Republic of Congo, Guatemala, Kenya, Pakistan, and Zambia. Current Problems in Diagnostic Radiology. 2017; 46(3): 210-5.

[10] Liberman AL, Newman-Toker DE. Symptom-Disease Pair Analysis of Diagnostic Error (SPADE): a conceptual framework and methodological approach for unearthing misdiagnosis-related harms using big data. BMJ Quality \& Safety. 2018; 27(7): 557-66.

[11] Shimkhada R, Solon O, Tamondong-Lachica D, Peabody JW. Misdiagnosis of obstetrical cases and the clinical and cost consequences to patients: a cross-sectional study of urban providers in the Philippines. Global Health Action. 2016; 9(1): 32672.

[12] Royce CS, Hayes MM, Schwartzstein RM. Teaching critical thinking: a case for instruction in cognitive biases to reduce diagnostic errors and improve patient safety. Academic Medicine. 2019; 94(2): 187-94.

[13] Mamede S, Hautz WE, Berendonk C, Hautz SC, Sauter TC, Rotgans J, Zwaan L, Schmidt HG. Think twice: effects on the diagnostic accuracy of returning to the case to reflect upon the initial diagnosis. Academic Medicine. 2020; 95(8): 1223-9.

[14] World health organization. Health services coverage statistics: antenatal care Coverage (percentage). Switzerland. 2012.

[15] Abebe S, Alemayehu A, Gebremariam A, Dirar A. Quality of antenatal care service in public health facilities of Chencha District, Gamo Gofa Zone, Southern Ethiopia. MOJ Women's Health. 2017; 4(3): 00086.

[16] Njoroge EW. The maternal and fetal outcomes among women with obstetric emergencies referred to the Kenyatta National Hospital, Nairobi, Kenya (Doctoral dissertation, University of Nairobi, Kenya).

[17] National Council for Population and Development (NCPD) and UNFPA. Kenya Country Office, Kenya Population Situation Analysis. Nairobi: NCPD. and UNFPA. 2013.

[18] Ministry of Health [Kenya]. Saving Mothers lives. First confidential report into maternal deaths in Kenya. 2017.

Kajiliwa M., Kamau G. Kenyans pay dearly for medical laxity. Standard Newspaper. 2017.

[19] World Health Organisation. Maternal mortality, sustainable development goals and global strategy for Women, children and adolescents' Health? 2018.

[20] Gacheri A. Tackling high maternal deaths in Kenya. Policy Brief, Parliament of Kenya. 2016.

[21] Kenya National Bureau of Statistics. The 2019 Kenya Population and Housing Census: Population by County and Sub-county. Kenya National Bureau of Statistics; 2019.

[22] District Health Information System. Ministry of Health. Diagnostic Errors. Republic of Kenya. 2017.

[23] Kothari C, Garg G. Research methodology: Methods and Techniques. Third edit. ed. New age international (p) limited, publishers. 2014.

[24] Kar Chatterjee S, Ray K, Das AK. Gap analysis between provisional diagnosis and final diagnosis in government and private teaching hospitals: a record-linked comparative study. Journal of Family Medicine and Primary Care. 2016; 5(3): 637.

[25] Chattopadhyaya A, Ghosh A, Chattopadhyay S, Sengupta S. Stator Current Harmonic Assessment of Induction Motor for Fault Diagnosis 1. 2013.

[26] Yego F, Williams JS, Byles J, Nyongesa P, Aruasa W, D'Este C. A retrospective analysis of maternal and neonatal mortality at a teaching and referral hospital in Kenya. Reproductive Health. 2013; 10(1): 1-8.

[27] Singh H. Helping organizations with defining diagnostic errors as missed opportunities in diagnosis. Jt Comm J Qual Patient Saf. 2014; 40(99): 102.

[28] Ordi J, Castillo P, Garcia-Basteiro AL, Moraleda C, Fernandes F, Quintó L, Hurtado JC, Letang E, Lovane L, Jordao D, Navarro M. Clinico-pathological discrepancies in the diagnosis of causes of death in adults in Mozambique: a retrospective observational study. PLoS One. 2019; 14(9): 0220657.

[29] Carayon P, Schoofs H, Karsh BT, Gurses AP, Alvarado CJ, Smith M, et al. Ex-worker accuses hospital of misdiagnosis after going blind and deaf, the star newspaper of 3rd January 2018. Available from: https//www.the-star.co.ke.

[30] Abimanyi-Ochom J, Bohingamu Mudiyanselage S, Catchpool M, Firipis M, Wanni Arachchige Dona S, Watts JJ. Strategies to reduce diagnostic errors: a systematic review. BMC medical informatics and Decision Making. 2019; 19(1): 1-4.

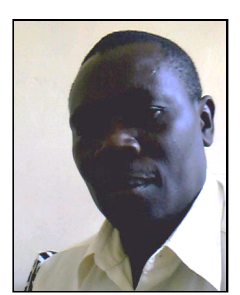

Wafula Nandebe David is a renowned Clinician with a keen interest in diagnosis and Medical Teacher at Kenya Medical Training College, Nairobi Department of Clinical Medicine. 